\title{
Polystyrene inverseopal tip as a solution indicator
}

\author{
Tonghua $\mathrm{Wu}^{1}$, Xiaohan $\mathrm{Su}^{1}$, Dongmei Zhao ${ }^{2 \mathrm{a}}$, Yanhong Zhang ${ }^{1 \mathrm{~b}}$, Liguo Sun ${ }^{1 \mathrm{c}}$ \\ ${ }^{1}$ Key Laboratory of Chemical Engineering Process \& Technology for High-efficiency Conversion, \\ College of Heilongjiang Province, Heilongjiang University, Harbin, 150080, China
}

${ }^{2}$ Department of Food and Environment Engineering, Heilongjiang East University, Harbin, 150086, China

\author{
azhaodongmei0322@aliyun.com(Corresponding Author), \\ bzyh3105@163.com (Corresponding Author), \\ csunliguo1975@163.com(Corresponding Author)
}

Keywords: Polystyrene; inverseopal tip; solution indicator; optical method

\begin{abstract}
An optical method was developed to monitor solubility of the polymer by taking advantage of reflection spectrum change of the dissolution tunable inverse opal photonic crystal. The dissolution tunable photonic bands of the polymer inverse opal photonic crystal were traceable to the chain motion of macromolecules. By observing the reflection spectrum change of the polystyrene inverse opal tip during solvent treatment, solubility of polystyrene was gotten.
\end{abstract}

\section{Introduction}

Since 1987 Yablonovitch and John brought forward the concept of photonic band gap (PBG), photonic crystal (PC) has aroused great interests of the scientists, the theories and preparation of which have flowered in the past twenty years[1,2]. Meanwhile, the applications of PC have spread many fields such as optical filters, optical valves, fibers, tunable laser, sensors, displays and storages[3-5]. Herein we put forward a new application of PC which made of polystyrene (PS) thin inverseopal film to be as a solution indicator. The solution of polymer is a complex progress to be describe, the promote method is to measure the degree of solution. In this study, we used silicon dioxide colloidal crystals as template to prepare ordered PS iverseopal film, then put them into the mixed solvent of ethanol $(\mathrm{EtOH})$ and acetone $\left(\mathrm{C}_{3} \mathrm{H}_{6} \mathrm{O}\right)$ and the proportion of $\mathrm{EtOH} / \mathrm{C}_{3} \mathrm{H}_{6} \mathrm{O}$ is 2:1,3:1,4:1, pure EtOH.

\section{Experimental Section}

Materials. Polystyrene were purchased from Sinopec Yangzi Petrochemical Company Ltd. Monodispersed silica microspheres were prepared by modified the Stöber method.

Preparation of polystrene inverse opal. Firstly, silica opal template with narrow reflection peak was formed via vertical deposition. Secondly, moderate melted polymer was infiltrated into air voids of the silica opal template, then the complex was cooled to $25{ }^{\circ} \mathrm{C}$ in room temperature condition. Thirdly, remove the glass substrate by soaking in water and etch the silica opal template with a $5 \mathrm{wt} \% \mathrm{HF}$ aqueous solution for half an hour. Lastly, residual HF was washed away with distilled water and the sample was dried under $\mathrm{N}_{2}$ atmosphere.

Solubility test. The film was cut into $3 \times 3 \mathrm{~mm}^{2}$ tips and put into the the solvent respectively and after definite time and measure the reflectance. The thin film put into the pure $\mathrm{C}_{3} \mathrm{H}_{6} \mathrm{O}$ became ropy and can not be get out after 30 seconds. And the thin film put into pure EtOH the reflectance 559nm after $2 \mathrm{~h}$, and others solution status as the fig 1 shows. So we can asseverate that pure $\mathrm{C}_{3} \mathrm{H}_{6} \mathrm{O}$ is well solvent of PS, the deliquescent of mixed solvent of EtOH and $\mathrm{C}_{3} \mathrm{H}_{6} \mathrm{O}$ descends when the percent of EtOH ascends, and the EtOH is not well one but it can swell PS a certain extent.To sum up, in this paper, we have given a simple and intuitionistic approach to estimate deliquescent property of polymer in solvent

Measuremnet. An optical microscope equipped with a fiber optic spectrometer 
(HR2000CG-UV-NIR, Ocean optics Inc.) was employed to detect reflection spectra. Microstructure images were obtained on a scanning electron microscopy (HITACHI, S-3000N) operated at $5.0 \mathrm{kV}$. Photographs of films were taken by an optical microscope (OLYMPUS BX51) equipped with a CCD camera (Media Cybernetics Evolution MP 5.0). Tg of bulk PS were measured by DSC (Pyris $1)$.

\section{Results and Discussion}

To get high-quality colloidal crystals, colloidal dispersions with suitable density and clean glass slide were of equal importance. The silica microspheres obtained were treated by centrifugation and ultrasonic dispersion to remove impurities and to ensure that silica microspheres were homogeneously dispersed in ethanol. The nitrogen was used to avoid dust contamination to the glass slide and further to alcohol colloid of microspheres in the process of treatment. Throughout the assembly process, the assembly unit was always in the stable air flow, invariable temperature, no vibration environment to obtain high quality silica coloidal crystals. After assembly, uniform silica coloidal crystal membrane was laid on glass slide. With three-dimensional ordered periodic structure, colloidal crystal would produce a strong reflection when light with particular wavelength irradiated on the surface of crystal. The wavelength of reflected light could be predicated by Bragg law. When the wavelength of light falled on the visible region, bright color could be exhibited. So the quality of colloidal crystals with the reflected light in the visible region could be determined by the brightness and uniformity of the color.
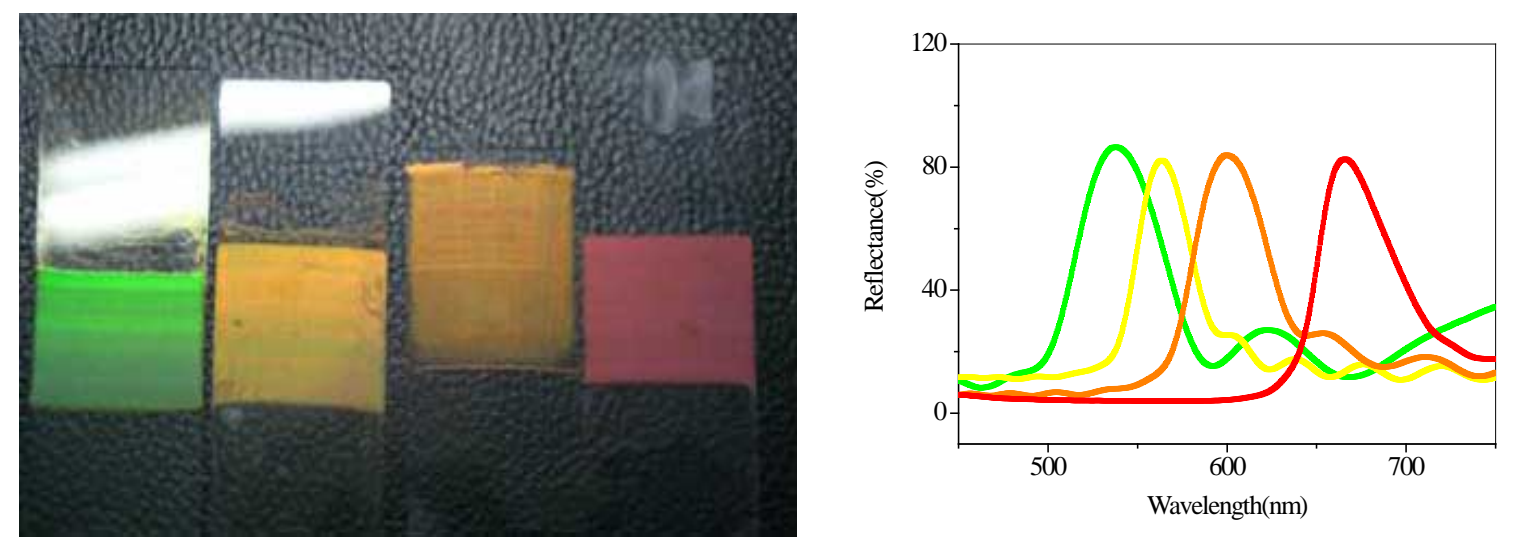

Fig.1 Photograph of silica opals on glass slide(left) and their reflectance spectra(right)

Fig. 1 was the digital photo of Sintered silica colloidal crystal membrane and the corresponding reflectance spectra. Sintered colloidal crystals exhibited bright, uniformly red and narrow reflection peaks, which indicated the high quality of colloidal crystals were prepared. In order to enhance contact degree of the silica microspheres and glass substrate, we used the method of heat treatment to the colloidal crystal template. The uniform heating rate from room temperature to $600{ }^{\circ} \mathrm{C}$ for $2 \mathrm{~h}$ was ensured in the process of heat treatment and kept $600{ }^{\circ} \mathrm{C}$ for an hour $(1 \mathrm{~h})$, then naturally cooled to room temperature to avoid the sudden drop. 

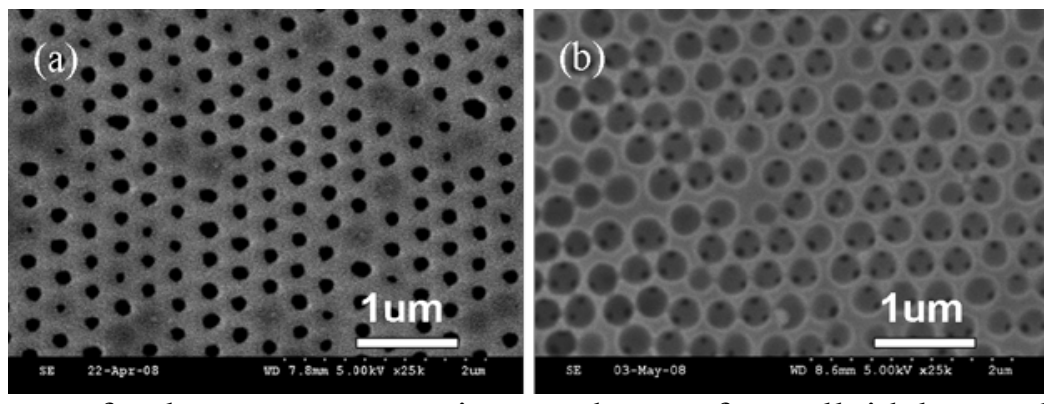

Fig.2 SEM images of polystyrene composite membrane after colloidal crystal templates were etched. (a ) colloidal crystal template without thermal treatment, (b) colloidal crystal template thermal treatment at $600{ }^{\circ} \mathrm{C}$.

The softening temperature of glass substrate was lower than the silica microspheres. When an appropriate heat treatment temperature was forced on the template the glass substrate would be soften while silica microspheres remained stable. So the silica microspheres on the bottom were embedded in the glass substrate, which greatly increased the contact area between the microspheres and the substrate. Fig.2 gave the SEM images of porous polystyrene membrane after colloidal crystal templates were etched. The ordered porous structure could be seen from Fig.2(b). Pore size was also increased significantly to approximate $300 \mathrm{~nm}$. It was showed that the silica colloidal crystal microspheres and glass substrate were combined closely, which provided a foundation to improve adhesion force between microspheres and substrate.

Fig. 3 showed the reflectance spectra change when proportion of the solvents, ethanol increase, the shift of the reflectance spectra decrease when time longer.
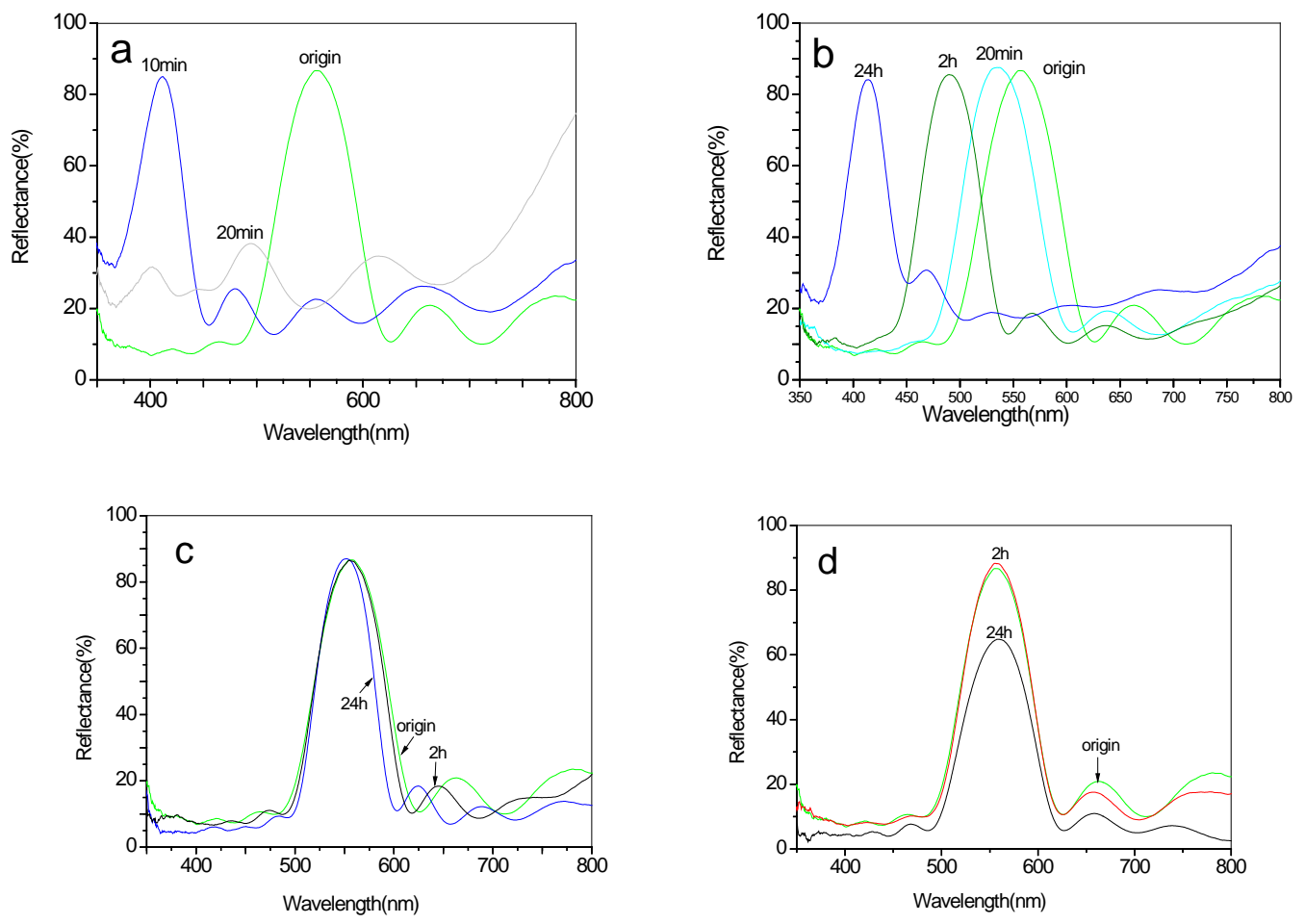

Fig.3 Reflectance spectra of PS inverseopal tips in mixed solvent $\left(\mathrm{EtOH} / \mathrm{C}_{3} \mathrm{H}_{6} \mathrm{O}=\right.$ 2:1(a),3:1(b),4:1(c), pure EtOH(d))

\section{Conclusion}

We reported an optical method to measure solubility of the polymer by taking advantage of the reflection spectrum change of the solution tunable inverseopal tips. As the color changes of the inverseopal tip were so obvious, the solubility could be directly estimated by naked eyes. The present study exploited a simple and effective way to measure the solubility of the polymer. 


\section{Acknowledgments}

The study has been supported by China post doctor foundition(2013M531008) and Heilongjiang Provincial Department of Education (12531521).

\section{References}

[1] E. Yablonovitch: Physical Review Letters Vol. 58(1987), p. 2059

[2] S. John: Physical Review Letters Vol. 58(1987), p. 2486

[3] Z. Z. Gu, H. Uetsuka, K. Takahashi, et al: Angew. Chem. Int. Ed. Vol. 42 (2003), p. 894

[4] Z. Z. Gu, R. Horie, S. Kubo, et al: Angew. Chem. Int. Ed. Vol. 41(2002), p. 1153

[5] L.G. Sun, Z. Y. Xie, H. Xu, et al: J. Nanosci. Nanotechnol. 12(2012), p. 1984 